\title{
Upscaling ice crystal growth dynamics in snow: Rigorous modeling and comparison to 4D X-ray tomography data
}

\author{
Q. Krol, H. Löwe* \\ WSL Institute for Snow and Avalanche Research SLF, 7260 Davos Dorf, Switzerland
}

\begin{abstract}
Presently a unified treatment of microstructure dynamics in terrestrial snow from principles of ice crystal growth is hindered by the lack of models for the evolution of the bicontinuous ice matrix. To this end we developed a rigorous microstructure upscaling scheme which is based on common pore-scale (vapor diffusion) principles of crystal growth to predict the averaged evolution of the interface morphology. We derived a coupled set of evolution equations for the (volume averaged) ice volume fraction, surface area per unit volume, Gaussian curvature and first and second moment of the mean curvature distribution and demonstrate their correctness by a comparison to interface tracking of idealized grains. In a second step we use the model as a benchmark tool without a-priori assumptions for a comparison to experiments of snow microstructure evolution via image analysis on 4D X-ray tomography data. The benchmarking allows quantifying uncertainties from local estimates of crystal growth velocities. Finally we demonstrate how the rigorous model facilitates a statistical assessment of common growth laws by combining 4D microstructure data with finite element numerics. The results show that the prediction of the surface area evolution from first principles demands further conceptual insight from ice crystal growth.

Keywords: Microstructure, Theory and modeling, Crystal growth, X-ray computed tomography, Porous material,
\end{abstract}

*Corresponding author

Email address: loewe@slf.ch (Q. Krol, H. Löwe)

This document is the accepted manuscript version of the following article:

Krol, Q., \& Löwe, H. (2018). Upscaling ice crystal growth dynamics in snow: rigorous modeling and comparison to 4D X-ray tomography data. Acta Materialia, 151, 478-487. https://doi.org/10.1016/j.actamat.2018.03.010 


\section{Introduction}

Snow on the ground is an interesting example of a porous, high-temperature material close to the melting point of ice, where a continuous evolution of microstructure, referred to as snow metamorphism [1], is ubiquitous under ambient conditions. The dynamical evolution originates from micro-scales where sintered ice crystals grow collectively from the vapor phase. Ice crystal growth is therefore not only of interest in view of the fascinating morphologies of isolated crystals [2, 3], it also constitutes the essential ingredient for upscaled material models of snow. There is an increasing demand to move from empirical to firstprinciples approaches of microstructure evolution to limit uncertainties of snow models in environmental or climate applications.

The prediction of the morphological evolution of snow on the ground is subject to the common difficulty of separation of spatial scales in heterogeneous materials [4]. Various techniques [5] can be employed to bridge microscopic (grain or pore) growth processes and macroscopic (spatially averaged) scales of interest for continuum modeling. However, snow microstructure evolution is still based on simple geometrical shapes when relating size changes to dominant non-equilibrium driving conditions (e.g. [6, 7, 8]). Existing approaches are based on variants of classical coarsening models [9] as a multi-particle diffusion problem, mediated by the curvature dependence of the equilibrium vapor pressure in the Gibbs-Thomson boundary condition.

Sphere-based approaches are however not satisfying for obvious reasons. The simplistic model geometry reflects by no means the complex, bicontinuous microstructure of snow. Recent advancements [10, 11, 12, 13, 14] in observing snow microstructure evolution via time-lapse experiments with X-ray micro-computed tomography ( $\mu \mathrm{CT})$ cannot be related to these approaches which hinders a full exploitation of advanced morphological observations. Tomography has e.g. revealed that the Euler characteristic is correlated with the evolution of the surface area of snow in uniaxial compression experiments [12]. Another study [11] has shown that the distribution of mean curvatures in snow develops morphological differences between growth and sublimation sites as a consequence of asymmetric surface kinetics. Even 
though a visual inspection of snow unambiguously demonstrates that kinetic effects play a role [1], it is difficult to quantitatively relate these aspects to averaged morphological properties. In addition, state-of-the-art 3D modeling of growing single crystals has demonstrated the importance of combining vapor and heat diffusion with anisotropic growth laws to reflect the symmetry of the crystal lattice [3, 15]. Presently, this insight is however completely disconnected from snow continuum modeling. Only a faithful representation of geometry will allow for the utilization of local thermodynamic aspects of ice crystal growth for terrestrial snow and exploit 4D data of snow evolution for improvements in upscaled snow models.

Apart from snow, the evolution of bicontinuous microstructures is relevant for other materials as well. A fundamental understanding of isothermal coarsening of bicontinuous structures can be obtained from phase field simulations [16]. The results of these simulations show that the joint probability distributions of principal curvatures is a suitable generalization of a size distribution to assess dynamic scaling in coarsening under conserved and non-conserved dynamics for arbitrary microstructures. A key difficulty in bicontinuous coarsening arises from topological changes which, in turn, affect the evolution of shape. The simulations from [17 indicate that a decay of the averaged mean curvature can only be explained if non-local terms of the growth velocity involving its surface Laplacian are taken into account. This kind of interface analysis highly benefits from a combination with 4D tomography measurements which has been demonstrated for the solidification of $\mathrm{Cu}-\mathrm{Al}$ composites [18]. However a full understanding of the coupled evolution of interface parameters in evolving microstructures is still missing and advancements in relating $4 \mathrm{D}$ data to rigorous evolution models is therefore of broader interest.

A common question in upscaling is the choice of parameters for which evolution equations must be derived. From a thermodynamic view point, upscaled conservation equations for mass and energy in snow [19] explicitly involve the ice volume fraction and the surface area per unit volume. Hence an appropriate microstructural viewpoint must at least provide equations for these quantities. However, it is often necessary to extend the set of parameters, either to ensure closure for the initial ones, or because there is specific interest in them. 
Recent work [20] has e.g. shown that the second moment $\overline{H^{2}}$ of the mean curvature distribution is of key relevance for snow since it controls the expansion of the two-point correlation function and the latter can be related to macroscopic transport properties via bounds [4]. Thus the dynamics of $\overline{H^{2}}$ also allows constraining the evolution of two-point correlations. This can be exploited in situations where full 3D microstructure observations are not available and the dynamical characterization relies on e.g. the estimation of interfacial shape via small-angle scattering [21].

A powerful theoretical approach to microstructure evolution was suggested by [22] who developed a mathematically rigorous description of averaged properties of an evolving interface by starting from the differential equations for the local, principal curvatures. Thereby, the averaged evolution of the surface is determined by the local interface velocity which must be provided as a "constitutive law" as closure relation. The strength of the approach [22] has been demonstrated e.g. by [18, 23].

The goal of the present paper is to build on [22] and derive a rigorous upscaling scheme for snow microstructure which can be coupled to upscaling of heat and mass transfer [19]. This is achieved by volume averaging the time evolution of local geometrical properties of the ice-air interface which is determined by a normal interface velocity relating crystal growth to geometrical and thermodynamic quantities. For snow-specific reasons, we generalize [22] to include a contribution of the interface velocity from mechanical deformation, and include an equation for the second moment of mean curvature. The averaged microstructural properties are governed by partial differential equations that are valid in a Eulerian frame of reference. We demonstrate the correctness of the equations in the absence of deformations by numerically tracking the interfaces of idealized grains under a prescribed growth law. This test enables us to infer numerical uncertainties that originate from the smoothness of the interface. In a next step we apply the same procedure to $4 \mathrm{D}$ data of a time-lapse snow metamorphism experiment under a stationary temperature gradient conducted by $\mu \mathrm{CT}$. This comparison quantifies the uncertainties of velocities estimates from 4D data and the representation of curvatures. Finally we exploit the analytical framework to statistically assess 
the relevance of common closure models. Using Finite Element simulations of heat transfer we provide evidence that a diffusion-limited picture is in contradiction to the experimentally observed evolution of the specific surface area.

The paper is organized as follows. In Section 2 we derive the microstructural evolution equations in the general setting. In Section 3 we simplify the equations to the situation of absent macroscopic gradients and show the correctness of the equations for idealized grains. In Section 4 we apply the same framework to 4D tomography data of snow evolution experiments and discuss the uncertainties. In Section 5 we show how the exact model can be used for a statistical assessment of growth laws. The results are discussed in Section 6 .

\section{Upscaling of microstructure in snow metamorphism}

\subsection{Preliminaries and notation}

We consider snow in a sample volume $V=V_{i}+V_{v}$ comprising ice and (vapor-filled) air sub-volumes $V_{i}, V_{v}$ separated by an interface $\Gamma$ with area $A=|\Gamma|$. The microstructure is characterized by an indicator function

$$
\mathcal{I}(\boldsymbol{x}, t)= \begin{cases}1 & \text { if } \boldsymbol{x} \in V_{i} \text { at time } t \\ 0 & \text { otherwise }\end{cases}
$$

The dynamical evolution of the indicator function can be inferred from the motion of the ice-air interface which is described by the discontinuous variant [22] of the level-set equation 24

$$
\frac{\partial}{\partial t} \mathcal{I}(\boldsymbol{x}, t)+\boldsymbol{w}(\boldsymbol{x}, t) \cdot \boldsymbol{\nabla} \mathcal{I}(\boldsymbol{x}, t)=0
$$

in terms of the total interface velocity field $\boldsymbol{w}$. Due to the relevance of both, we allow for a contribution $\boldsymbol{u}$ from mechanical deformations and $\boldsymbol{v}$ from crystal growth according to

$$
\boldsymbol{w}=\boldsymbol{u}+\boldsymbol{v}
$$

Only the growth part can be assumed to be normal to the interface, viz. $\boldsymbol{v}=v_{n} \boldsymbol{n}$ in terms of

the normal vector $\boldsymbol{n}$. Eq. (2) is defined in the sense of distributions since $\nabla \mathcal{I}$ is a gradient of 
a discontinuous function that acts as delta function concentrated on the interface according to

$$
\nabla \mathcal{I}=-\boldsymbol{n} \frac{\partial \mathcal{I}}{\partial n}
$$

where $\partial \mathcal{I} / \partial n$ is a $1 \mathrm{D}$ delta function in normal direction. Note, with this definition the normal vector field is oriented from ice to air. The local mean curvature $H$ and the Gaussian curvature are given by the spatial derivatives of the normal vector

$$
H=\frac{1}{2} \nabla \cdot \boldsymbol{n}=\frac{1}{2}\left(\kappa_{1}+\kappa_{2}\right), \quad K=\kappa_{1} \kappa_{2}
$$

where $\kappa_{1}$ and $\kappa_{2}$ denote the principal curvatures.

\subsection{Volume and surface averages}

The volume average of a function $f(\boldsymbol{x})$ for $\boldsymbol{x} \in V$ is defined by

$$
\langle f\rangle:=\frac{1}{V} \int d \boldsymbol{x} f(\boldsymbol{x}) .
$$

A function $f$ can be restricted to the ice or vapor phase via $f_{\mathrm{i}}:=f \mathcal{I}$ or $f_{\mathrm{v}}:=f(1-\mathcal{I})$ and the volume-average of $f_{\mathrm{i}}$ can be written as

$$
\left\langle f_{\mathrm{i}}\right\rangle=\phi \frac{1}{V_{i}} \int_{V_{\mathrm{i}}} f(\boldsymbol{x}) d \boldsymbol{x}=: \phi\langle f\rangle_{\mathrm{i}}
$$

which defines the intrinsic average $\langle\bullet\rangle_{i}$ and the ice volume fraction $\phi=\langle\mathcal{I}\rangle=V_{i} / V$.

Due to the delta-function in the level-set equation, volume-averages of various microstructural quantities reduce to surface averages. For a function $g$ defined on the interface $\Gamma$ the surface average is defined by

$$
\bar{g}:=\frac{1}{s}\left\langle g(\boldsymbol{x}) \frac{\partial \mathcal{I}}{\partial n}\right\rangle=\frac{1}{A} \int_{\Gamma} \operatorname{dag} g(\boldsymbol{x})
$$

with the surface area element $d a$ and the surface area per unit volume

$$
s=\left\langle\frac{\partial \mathcal{I}}{\partial n}\right\rangle=\frac{A}{V} .
$$

Using the above notation, we can restate the temporal and spatial averaging theorems which are required to relate averages of gradients to gradients of averages. When calculating the 
time derivative of Eq. (6) and exploiting the level-set equation Eq. (2), additional surface average terms arise for time and space derivatives of a phase-restricted functions $f_{i}$ according to

$$
\frac{\partial\left\langle f_{i}\right\rangle}{\partial t}=\left\langle\frac{\partial f}{\partial t}{ }_{i}\right\rangle+s \overline{f w_{n}}
$$

in terms of the normal component of the interface velocity field $w_{n}$, and according to

$$
\boldsymbol{\nabla}\left\langle f_{i}\right\rangle=\left\langle\boldsymbol{\nabla} f_{i}\right\rangle-s \overline{f \boldsymbol{n}} .
$$

These equations are also known by the volume and surface averaging theorems [26].

\subsection{Evolution of the ice volume fraction}

The micro-scale ice mass conservation equation can be written as

$$
\frac{\partial \rho_{\mathrm{i}}}{\partial t}+\nabla \cdot\left(\rho_{\mathrm{i}} \boldsymbol{u}_{\mathrm{i}}\right)=0
$$

where $\boldsymbol{u}_{\mathrm{i}}=\boldsymbol{u} \mathcal{I}$ is the ice deformation velocity field and $\rho_{i}=\rho \mathcal{I}$ the density, both restricted to the ice phase. Note that $\left\langle\rho_{i}\right\rangle=\phi \rho_{\text {ice }}$ with $\rho_{\text {ice }}$ the density of ice.

In principle $\boldsymbol{u}$ must be determined by a micro-scale mechanical treatment (force balance and constitutive equation). In the following we shall assume these quantities to be given. By volume averaging the continuity equation Eq. 12 and using the averaging theorems Eq. (10) and Eq. (11) we obtain

$$
\frac{\partial \phi}{\partial t}+\nabla\left(\phi\langle\boldsymbol{u}\rangle_{i}\right)=s \overline{v_{\mathrm{n}}} .
$$

This result corresponds to [19] when $\boldsymbol{u}$ is set to zero. It relates ice mass changes in the control volume $V$ to the deformation velocity $\boldsymbol{u}$ and the accretion of mass via $v_{n}$ if integrated over the surface $s$.

\subsection{Evolution of the surface area per unit volume}

Eq. (13) implies that the macroscopic mass conservation equation requires an evolution equation for $s$, the surface area per unit volume. Note, that $s$ differs from the surface area per ice volume that is commonly termed specific surface area in the context of snow. 
Due to the general form of the velocity field (3), the derivation of the surface area evolution must generalize the approach from [22]. The essential steps are provided in Appendix 8.1 . the result is given by

$$
\frac{\partial s}{\partial t}+\nabla s \overline{\boldsymbol{w}}=s \overline{\mathcal{F}_{s}}
$$

in terms of the source term $\mathcal{F}_{s}$ that is defined by

$$
\mathcal{F}_{s}=2 v_{n} H+(\boldsymbol{\nabla} \cdot \boldsymbol{u})-\boldsymbol{n}[\boldsymbol{\nabla} \otimes \boldsymbol{u}] \boldsymbol{n} .
$$

Here $\otimes$ denotes the outer product of two vectors. Eq. (14) is a partial differential equation for the surface area per unit volume in a Eulerian frame of reference which accounts for the change in surface area from the divergence in the mean velocity field (left hand side) while the right hand side captures the contribution from growth (first term) and the first order correction of the surface area from deformation gradients involving $\boldsymbol{u}$ (second and third term). Due to a typo in [22] the first term on the right hand side of (15) differs by a factor of 2 which affects the form of the following curvature equations as well.

\subsection{Evolution of interfacial curvatures}

While the evolution equation for the ice volume fraction (13) requires the surface area, the equation for the surface area (15) requires the mean curvature. Analogous to [22] the local evolution equations for arbitrary moments of the curvature can be derived again by algebraic manipulation of (2) and subsequent averaging. For the curvatures we neglect higher order changes from non-normal components of the interface velocity via $\boldsymbol{u}$ in (3) which is tedious, but principally feasible via the evolution of the metric tensor [27]. The key steps are provided in the Appendix 8.2, resulting in the set of continuity equations

$$
\begin{aligned}
\frac{\partial}{\partial t} \bar{H}+\bar{w} \cdot \boldsymbol{\nabla} \bar{H} & =-\frac{1}{s} \boldsymbol{\nabla} s \overline{\boldsymbol{w}(H-\bar{H})}+\overline{\mathcal{F}_{H}}+\overline{(H-\bar{H}) \mathcal{F}_{s}} \\
\frac{\partial}{\partial t} \bar{K}+\bar{w} \cdot \boldsymbol{\nabla} \bar{K} & =-\frac{1}{s} \boldsymbol{\nabla} s \overline{\boldsymbol{w}(K-\bar{K})}+\overline{\mathcal{F}_{K}}+\overline{(K-\bar{K}) \mathcal{F}_{s}} \\
\frac{\partial}{\partial t} \overline{H^{2}}+\bar{w} \cdot \boldsymbol{\nabla} \overline{H^{2}} & =-\frac{1}{s} \boldsymbol{\nabla} s \overline{\boldsymbol{w}\left(H^{2}-\overline{H^{2}}\right)}+2 \overline{\mathcal{F}_{H}}+\overline{\left(H^{2}-\overline{H^{2}}\right) \mathcal{F}_{s}}
\end{aligned}
$$


with the source terms

$$
\begin{aligned}
& \mathcal{F}_{H}=-\left(2 H^{2}-K\right) v_{n}-\frac{1}{2}\left(\partial_{1}^{2}+\partial_{2}^{2}\right) v_{n} \\
& \mathcal{F}_{K}=-2 H K v_{n}-\left(\kappa_{2} \partial_{1}^{2}+\kappa_{1} \partial_{2}^{2}\right) v_{n} .
\end{aligned}
$$

Here $\partial_{1}^{2}, \partial_{2}^{2}$ denote the second derivatives along the principal directions on the surface with corresponding principal curvatures $\kappa_{1}=H+\sqrt{H^{2}-K}$ and $\kappa_{2}=H-\sqrt{H^{2}-K}$. Eqs. (13), (15), (16), (17), (18) constitute the evolution equations for the most important interface parameters hitherto investigated for snow.

\subsection{Reduced evolution equations}

For single grains or statistically homogeneous microstructures the evolution equations can be further simplified due to vanishing gradient terms in Eqs. (16)-(18). Furthermore we can neglect deformation in the evolution for the averaged surface area, i.e. the last two terms in $\mathcal{F}_{s}$ vanish in $(15)$. Overall we end up with

$$
\begin{aligned}
\frac{\partial s}{\partial t} & =2 s \overline{v_{n} H} \\
\frac{\partial \bar{H}}{\partial t} & =\overline{v_{n} K}-2 \bar{H} \overline{v_{n} H}-\frac{1}{2} \overline{\left(\partial_{1}^{2}+\partial_{2}^{2}\right) v_{n}} \\
\frac{\partial \bar{K}}{\partial t} & =-2 \overline{v_{n} H} \bar{K}-\overline{\kappa_{2} \partial_{1}^{2} v_{n}}-\overline{\kappa_{1} \partial_{2}^{2} v_{n}} \\
\frac{\partial \overline{H^{2}}}{\partial t} & =-2 \overline{v_{n} H^{3}}+2 \overline{v_{n} H K}-2 \overline{H^{2}} \overline{v_{n} H}-\overline{H\left(\partial_{1}^{2}+\partial_{2}^{2}\right) v_{n}}
\end{aligned}
$$

as a minimal model for the microstructure evolution in snow.

\section{Interface evolution of idealized grains}

\subsection{Image analysis}

To provide confidence for the correctness of 20, all terms must be computed individually from 3D microstructures. To this end we employ Paraview 28, and its supplied VTK mesh algorithms. We exploit that applying Paraview's UnstructuredGradient filter to functions on meshed surfaces yields an approximation of the surface gradient $P \nabla$. Here $P$ denotes the orthogonal projection on the surface tangent space. Thus, by application to the normal vector field, we obtain the shape operator $P \boldsymbol{\nabla} \otimes \boldsymbol{n}$ which is diagonalized to obtain the 
principal directions $\boldsymbol{t}_{1}, \boldsymbol{t}_{2}$ as eigenvectors. The corresponding eigenvalues are the principal

curvatures $\kappa_{1}, \kappa_{2}$ from which all curvature terms in 20 can be computed. For a given time series of images, we computed the interface velocity field with the interface tracking method developed in [29]. The second-order surface derivatives of the interface velocity are computed from the surface Hessian $H\left(v_{n}\right)=P \boldsymbol{\nabla} \otimes P \boldsymbol{\nabla} v_{n}$ of the velocity via $\boldsymbol{t}_{i} \cdot H\left(v_{n}\right) \boldsymbol{t}_{i}, i=1,2$. As proof of principle, we have conducted a test on a sphere where we defined the interface velocity $v_{n}$ by the first non-trivial spherical harmonics and confirmed that the trace of $H\left(v_{n}\right)$ satisfies the well-known eigenfunction property of the surface Laplacian. We note that the present method of computing curvatures differs from the one previously used in [29, 20] which directly employs Paraview's curvature filter after interface smoothing. The present method was found to be less affected by surface roughness and conveniently exploits the required diagonalization to compute the surface gradients.

\subsection{Generation of initial states}

To assess the correctness of the coupled evolution equations in combination with the image analysis we first consider the evolution of grains with idealized shapes but non-trivial curvature distributions (spheroids, tori) under a uniform growth law and compare different ways to extract the interface.

To demonstrate the subtle influence of surface smoothness we created three equally sized spheroids based on different methods to generate the surface mesh. Spheroid A is based on a parametric surface from a vtkSphere object and rescaled to a spheroid using vtkTransform. Spheroid B was sampled on a voxel image and convoluted with a Gaussian filter (support 4.5, $\sigma=9$ voxels) for smoothing before applying a contour filter to obtain the triangulated surface mesh as the zero-level set of the image. This corresponds to the procedure that is later applied to the tomography data. Spheroid C is also based on a binary voxel image, but here the contour filter was applied before the mesh was smoothed via Laplacian smoothing with $S=500$ iterations, as described and previously used in [29]. To also include a grain with negative mean curvatures, a torus was chosen which is also based on a parametric surface with numerically exact positions of the mesh-points. All objects are shown in Fig. 1 
revealing the impact of surface extraction on the calculated mean curvatures.

\subsubsection{Results}

All objects are evolved forward in time by Euler integration of the trajectories of the mesh points under a uniform normal velocity field $v_{n}$ using the VTK filter WarpByVector. To ensure stability via the CFL-condition [24], time steps are adapted to the mesh size. The procedure yields a time series of surfaces and the averaged quantities $s, \bar{H}, \bar{K}, \overline{H^{2}}$ are obtained for each time-step by integrating their local counterparts over the surface according to (8). This allows comparing left and right hand side of the evolution equations (20) using a Crank-Nicholson scheme for their (finite-difference) discretization. In the following, we refer to the left hand side as the measured quantity and the right hand side as the modeled quantity. The results are shown in Fig. 2, For the Spheroid A and the torus, which are based on parametric surfaces, a very good agreement is obtained indicating the correctness of equations and calculation of interface parameters. The comparison of Spheroid A to B and C (Fig. 1) reveals the influence of different smoothing procedures when the objects are derived from voxel data, similar to the typical experimental situation discussed below. In particular higher order moments such as $\overline{H^{2}}$ are affected considerably by the apparent surface roughness (Fig. 2), though still resulting in a reasonable correlation between modeled and measured rates.

The results for the evolution of idealized grains show that under certain requirements of surface smoothness, the image analysis can be applied to infer the evolution of interface properties via (20). This comparison can be directly carried out at the level of their governing differential equations if an estimate of the interface velocity is available.

\section{Ice-air interface evolution from 4D tomography data}

\subsection{Time lapse experiments and interface tracking}

Next we conduct a comparison of the model to 4D image data of a temperature gradient metamorphism experiment by using interface tracking to estimate the a-priori unknown interface velocity [29]. To this end we analyzed two temperature gradient experiments (Series 
1 and 2) that were previously presented in [10]. In these experiments homogeneous, cylin-

drical snow samples of diameter $3.6 \mathrm{~cm}$ and height $2 \mathrm{~cm}$ were forced into a non-equilibrium state by applying a constant temperature gradient via Peltier elements in an instrumented tomography sample holder to monitor the microstructure by $\mu \mathrm{CT}$. The snow in the experiments has the same mean temperature $T=-8^{\circ} \mathrm{C}$, while slightly different temperature gradients $\nabla T=\{46,55\} \mathrm{K} / \mathrm{m}$ were applied for a duration of $\{384,665\} \mathrm{h}$ with a time step of $8 \mathrm{~h}$ between successive $\mu \mathrm{CT}$ scans.

For the image analysis the reconstructed binary images were blurred with a Gaussian filter (support $=2.0, \sigma=1.5)$ to improve the extraction of the zero-level set from the contour filter, as suggested by the performance for spheroid B from the previous section. The procedure described in Sec. 3.1 yields local estimates of the geometrical quantities $s, H, K, H^{2}, \kappa_{1}, \kappa_{2}$ and the interface velocity $v_{n}$ from which the right hand side ("modeled") of (20) can be computed by numerical integration over the surface. We have checked that the involved curvatures and velocities do not show a trend as a function of vertical position in the sample to justifiy the assumption of statistical homogeneity for neglecting the gradient terms in using (20). This cannot be expected to be generally valid since coarsening in low density snow is accompanied by non-zero deformation gradients [29, 12]. For a visual inspection, the initial $(t=0)$ and intermediate $(t=288 \mathrm{~h})$ states of the microstructure are shown in Fig. 3 and colored by their estimated interface velocities.

\subsection{Results}

The comparison of the measured and modeled rates from Eq. 20) (again via CrankNicholson finite differencing) is shown in Fig. 4. The volume fraction rates $\dot{\phi}$ allow for the most basic assessment of the derived interface velocities via the right hand side of (13) which highly underestimates the measured rates. This is partly caused by crystals entering and leaving the control volume during a time step, implying a considerable error on the velocity estimates. In addition, the interface tracking method converges to the correct values only for high temporal and spatial resolution [29]. Likewise, the modeled values for $\dot{s}$ are also significantly influenced by the errors of $2 \overline{v_{n} H}$. As a remedy for the other parameters, we 
have therefore replaced $2 \overline{v_{n} H}$ on the right hand side by the measured $\dot{s} / s$ values which can be estimated reliably. This is immediately reflected by a more reasonable agreement for $\dot{\bar{H}}$ in comparison to $\dot{s}$. We conducted a sensitivity run of the analysis without the surface

\section{Statistical analysis of crystal growth laws}

Independent of the errors in the estimation of interface velocities revealed in the previous section, the exact model enables a statistical assessment of crystal growth laws which typically depend on quantities that can be computed with higher accuracy.

\subsection{Growth laws for temperature gradient driven growth}

If crystal growth is modeled as a variant of a Stefan-type of diffusion problem [2], the local interface velocity must be related to the chemical potential difference between ice and vapor, i.e. to the difference between the actual vapor concentration $\rho_{v}(\boldsymbol{x})$ and the equilibrium concentration $\rho_{v}^{\mathrm{eq}}(\boldsymbol{x})$ at a point $\boldsymbol{x}$ on the interface according to $v_{n}(\boldsymbol{x})=\alpha\left[\rho_{v}(\boldsymbol{x})-\rho_{v}^{\mathrm{eq}}(\boldsymbol{x})\right]$ with a kinetic coefficient $\alpha$. It is well-known that the excess vapor concentration comprises diffusive and kinetic contributions [7], their relative importance was however difficult to assess quantitatively so far. If for the diffusion limited part local equilibrium between temperature and vapor is assumed [30], the local interface velocity can be related to temperature gradients in the pore space according to [20]

$$
v_{n}(\boldsymbol{x})=\left.A \boldsymbol{n}(\boldsymbol{x}) \cdot \nabla T(\boldsymbol{x})\right|_{+},
$$


where + denotes the limit of approaching the interface from the pore side.

To assess 21] we have computed the temperature fields for Series 1,2 in the microstructure by the Finite Element Method [10] using standard means of a voxel-based solution of is superimposed by more complex microstructural signatures in $v_{n}(H)$. The analyzed Series 2 provides clear evidence that the relation (21), which has been confirmed statistically [20], 
is merely a side effect and not the origin of the observed evolution of the surface area per unit volume since it is in contradiction with 22 .

To further demonstrate the morphological differences between the two series we compared the difference between sublimating and growing surface sites by constraining the surface averages on upward and downward faces as a proxy for it (cf. Fig. 3 and [1] ). The results are shown in Fig. 6] which confirms the findings from [11]: While $\bar{H}$ on growing and sublimating parts remain the same, $\overline{H^{2}}$ starts from the same initial values but splits up during the evolution reflecting the morphological asymmetry between growing and sublimating surfaces. The onset of disagreement found in Fig. 5 roughly corresponds to the onset of the $\overline{H^{2}}$ split up in Fig. 6. Fig. 6 also reveals that the width of the curvature distribution $\overline{H^{2}}-\bar{H}^{2}$ of Series 2 is considerably (60\%) higher when compared to Series 1.

\section{Discussion}

\subsection{Main findings}

We have derived a mathematically exact model for the coupled evolution of the most important parameters (the surface area and curvature moments up to second order $s, \bar{H}, \bar{K}, \overline{H^{2}}$ ) that are presently used to analyze snow microstructure in tomography experiments [31, 12, 11, 20]. The derivation is based on volume averaging the (discontinuous) level-set equation which is a full dynamical characterization of the ice indicator function at the micro-scale. The volume averaged evolution equations (13), (15), (16), (17), (18) enables us to bridge micro to macro scales which are relevant for continuum snow modeling [19].

We extended the approach from [22] to a contribution in the interface velocity stemming from mechanical deformations (cf. Eq. 15). Even though we have not yet exploited these terms in the analysis, they are relevant since crystal growth in snow is always accompanied by viscoplastic deformations. The growth contribution to the interface velocity originates from the non-equilibrium situation at the ice-air interface at the micro-scale, within the known limitations of diffusion modeling of crystal growth [2]. The set of derived equations must be regarded as the starting point of a hierarchy of evolution equations for the moments 
of the joint distribution of mean and Gaussian curvatures. However, the associated closure problem is irrelevant for a validation of the PDEs in differential form, since all terms can be computed solely from 4D observations without a priori information.

We have shown that the equations are formally correct in a minimal setting (Eq. (20) though uncertainties of the solution of the Laplace (heat) equation at the interface [29] on voxel data must be acknowledged. Here further insight in the relation between kinetic effects 
and shape of ice crystals is required. As a first step it seems necessary to clarify the role of emerging only at larger scales [32]. It seems thus feasible to combine the interface tracking

the morphological asymmetry between growing and sublimating parts of the surface (Fig. 6). The asymmetry reflects the observation that sublimating surfaces (upward, cf. 3) tend to be rounded while growing surfaces (downward) become faceted prior to the skeletal instability (onset visible in Fig. 3). This is consistent with a variance of the mean curvatures which is higher on the downward surfaces, confirming the observations from [11].

A sensitivity analysis of the minimal model has indicated that the surface derivatives in Eq. (20) are highly important for the prediction of the evolution of curvatures in bicontinuous systems undergoing topological changes. This is in agreement with previous results from [17] and demands new ideas to quantify the behavior of the surface gradients when sweeping through the singularity of a toplogical transition (e.g. pinch-off). The latter are notoriously difficult for surface tracking methods, while they are naturally accounted for in phase field models. The impact of surface gradients for topological transitions [17] can be illustrated by a simple reasoning. Upon pinch-off, the velocity field must develop a discontinuity at the tip, implying a delta-function for derivatives which may lead to a non-differentiable evolution of the averaged parameters $H, K, H^{2}$. For a finite number of droplets, the consequence of topology changes in the Mullins-Sekerka problem on a non-differentiable surface area is shown in [34]. Albeit not mathematically exact, topological changes may be captured on a phenomenological level as source terms in the evolution equations as suggested in [22].

\subsection{Limitations and prospects}

It is obvious that inferring ice crystal growth dynamics solely from image data is inherently ill-posed, since discriminating the cause for a moving ice-air interface either by deformation or growth is locally impossible: A single sphere growing in temperature gradient simply migrates in a shape-invariant way [30], equivalent to a rigid-body translation. The problem must thus be further constrained by additional micro-scale information on the strain field (densification). Fig. 3 reveals that growth is non-uniform over length scales of a single grain, while deformation processes are expected to be slow, with heterogeneities 
with e.g. volume correlation techniques on hierarchical scales and correct for deformation gradients that were neglected in (20). Presently, deformations hinder a benchmark analysis (Sec. 4) for isothermal coarsening experiments of low density snow [33]. These deformations

\section{Conclusions}

We have derived a mathematically exact upscaling model for microstructure parameters which are presently most commonly used to characterize snow microstructure in tomography experiments. The key ingredient is the local interface velocity of the ice-air interface 
which characterizes local crystal growth dynamics. The model can be used to benchmark the involved image analysis methods, since local estimates of the interface velocity can be obtained from 4D X-ray tomography image data without a-priori assumptions. Alternatively the model can be used for a statistical assessment of growth laws to improve simplifications for macroscopic continuum modeling of snow. Due to the rigorous starting point, we believe that the derived evolution scheme is a powerful tool to assess the simplifications employed for crystal growth in terrestrial snow by combining 4D data and simulations and exploiting the overlap to closely related coarsening problems.

\section{Acknowledgements}

This work was funded by SNSF through grant no. 200021_143839.

\section{Appendices}

\subsection{Derivation of the surface area equation}

To derive (14) we consider the auxiliary expression

$$
\frac{\partial}{\partial t}(\boldsymbol{n} \cdot \boldsymbol{\nabla} \mathcal{I})+\nabla \cdot([\boldsymbol{w} \otimes \boldsymbol{n}] \boldsymbol{\nabla} \mathcal{I})
$$

and note, by taking the gradient of (2), the validity of another auxiliary relation

$$
\frac{\partial}{\partial t} \boldsymbol{\nabla} \mathcal{I}+\boldsymbol{w}[\boldsymbol{\nabla} \otimes \nabla] \mathcal{I}=-[\boldsymbol{\nabla} \otimes \boldsymbol{w}] \boldsymbol{\nabla} \mathcal{I}
$$

Carrying out the derivatives in (23) using the product rule, subsequently inserting (24) and further exploiting $\dot{\boldsymbol{n}} \cdot \boldsymbol{n}=0$ and $(\boldsymbol{\nabla} \otimes \boldsymbol{n}) \cdot \boldsymbol{n}=\mathbf{0}$ yields the identity

$$
\frac{\partial}{\partial t}(\boldsymbol{n} \cdot \boldsymbol{\nabla} \mathcal{I})+\nabla \cdot([\boldsymbol{w} \otimes \boldsymbol{n}] \boldsymbol{\nabla} \mathcal{I})=(\boldsymbol{\nabla} \cdot \boldsymbol{w})(\boldsymbol{n} \cdot \boldsymbol{\nabla} \mathcal{I})-\boldsymbol{n} \cdot[\boldsymbol{\nabla} \otimes \boldsymbol{w}] \boldsymbol{\nabla} \mathcal{I}
$$

which is valid for arbitrary $\boldsymbol{w}$. Now we insert the specific superposition (3) into the right hand side of (25) and note that the two velocity contributions must be treated differently: For the deformation part $\boldsymbol{u}$ no further simplifications can be made. For the growth part 
$\boldsymbol{v}=v_{n} \boldsymbol{n}$ that is normal to the interface, the right hand side reduces to $v_{n}(\boldsymbol{\nabla} \cdot \boldsymbol{n})(\boldsymbol{n} \cdot \boldsymbol{\nabla} \mathcal{I})$.

After insertion of Eq. (14) and reshuffling terms we arrive at (16) which has been re-written in resemblance to [22] by means of $\overline{H \mathcal{F}_{s}}-\bar{H} \overline{\mathcal{F}_{s}}=\overline{(H-\bar{H}) \mathcal{F}_{s}}$. Equations for $\bar{K}$ and $\overline{H^{2}}$ can be derived accordingly.

$$
\dot{\kappa}_{i}=-v_{n} \kappa_{i}^{2}-\partial_{i}^{2} v_{n}
$$

The time evolution for $H, K, H^{2}$ can be inferred by using (5) which yields

$$
\dot{H}=\mathcal{F}_{H}, \quad \dot{K}=\mathcal{F}_{K}, \quad \dot{H}^{2}=2 H \mathcal{F}_{H}
$$

with $\mathcal{F}_{H, K}$ given by Eq. (19). The respective Eulerian counterparts of (28) are obtained by respectively adding $\boldsymbol{w} \cdot \boldsymbol{\nabla}(H), \boldsymbol{w} \cdot \boldsymbol{\nabla}(K), \boldsymbol{w} \cdot \boldsymbol{\nabla}\left(H^{2}\right)$ on the left hand side, e.g

$$
\dot{H}+\boldsymbol{w} \cdot \nabla(H)=\mathcal{F}_{H}
$$

The further derivation of averaged quantities is shown below for the mean curvature $\bar{H}$ as an example. To this end we multiply (26) by $H$ and add (29) times $\partial \mathcal{I} / \partial n$ which yields

$$
\frac{\partial}{\partial t}\left(H \frac{\partial \mathcal{I}}{\partial n}\right)+\nabla \cdot\left(\boldsymbol{w} H \frac{\partial \mathcal{I}}{\partial n}\right)=H \mathcal{F}_{s} \frac{\partial \mathcal{I}}{\partial n}+\mathcal{F}_{H} \frac{\partial \mathcal{I}}{\partial n}
$$

Applying volume averaging to (30) and using (8) in the form $\langle f \partial \mathcal{I} / \partial n\rangle=s \bar{f}$, we obtain

$$
\frac{\partial}{\partial t}(s \bar{H})+\nabla(s \overline{\boldsymbol{w} H})=s \overline{\mathcal{F}_{H}}+s \overline{H \mathcal{F}_{s}}
$$


[1] S. C. Colbeck, An overview of seasonal snow metamorphism, Reviews of Geophysics 20 (1) (1982) 45-61. doi:10.1029/RG020i001p00045.

[2] K. G. Libbrecht, The physics of snow crystals, Rep. Prog. Phys. 14 (4) (2005) 599-895.

[3] J. W. Barrett, H. Garcke, R. Nürnberg, Numerical computations of faceted pattern formation in snow crystal growth, Phys. Rev. E 86 (2012) 011604. doi:10.1103/ PhysRevE.86.011604.

[4] S. Torquato, Random heterogeneous materials: Microstructure and macroscopic Properties, Interdisciplinary Applied Mathematics, Springer, 2002.

[5] J. G. Berryman, Comparison of upscaling methods in poroelasticity and its generalizations, Journal of Engineering Mechanics 131 (9) (2005) 928-936. doi:10.1061/(ASCE) 0733-9399(2005) 131:9(928).

[6] E. E. Adams, R. L. Brown, A model for crystal development in dry snow, Geophys. Res. Lett. 9 (11) (1982) 1287-1289. doi:10.1029/GL009i011p01287.

[7] S. C. Colbeck, Theory of metamorphism of dry snow, Journal of Geophysical Research: Oceans 88 (C9) (1983) 5475-5482. doi:10.1029/JC088iC09p05475.

[8] L. Legagneux, F. Dominé, A mean field model of the decrease of the specific surface area of dry snow during isothermal metamorphism, J. Geophys. Res. Earth 110 (2005) F04011. doi:10.1029/2004JF000181.

[9] L. Ratke, P. Voorhees, Growth and coarsening, Springer, New York, 2002.

[10] B. R. Pinzer, M. Schneebeli, T. U. Kaempfer, Vapor flux and recrystallization during dry snow metamorphism under a steady temperature gradient as observed by time-lapse micro-tomography, The Cryosphere 6 (5) (2012) 1141-1155. doi:10.5194/ tc-6-1141-2012. 
[17] C.-L. Park, P. Voorhees, K. Thornton, Evolution of interfacial curvatures of a bicontinuous structure generated via nonconserved dynamics, Acta Materialia 90 (2015) 182 193. doi:http://dx.doi.org/10.1016/j.actamat.2015.02.037.

[18] J. Fife, J. Gibbs, E. Gulsoy, C.-L. Park, K. Thornton, P. Voorhees, The dynamics 
of interfaces during coarsening in solid-liquid systems, Act. Mater. 70 (2014) 66-78. doi:10.1016/j.actamat.2014.01.024.

[19] N. Calonne, C. Geindreau, F. Flin, Macroscopic modeling for heat and water vapor transfer in dry snow by homogenization, J. Phys. Chem. B 118 (47) (2014) 13393-13403, pMID: 25011981. doi:10.1021/jp5052535.

[20] Q. Krol, H. Löwe, Relating optical and microwave grain metrics of snow: the relevance of grain shape, The Cryosphere 10 (6) (2016) 2847-2863. doi:10.5194/tc-10-2847-2016.

[21] M. A. Micha, C. Burger, M. Antonietti, Generalized phase diagram for microphaseseparated systems and its determination by small-angle scattering, Macromolecules 31 (17) (1998) 5930-5933. doi:10.1021/ma980085c.

[22] D. A. Drew, Evolution of geometric statistics, SIAM Journal on Applied Mathematics 50 (3) (1990) 649-666. doi:10.1137/0150038.

[23] C. Morel, On the surface equations in two-phase flows and reacting single-phase flows, International Journal of Multiphase Flow 33 (2007) 1045-1073. doi:10.1016/j. ijmultiphaseflow.2007.02.008.

[24] J. A. Sethian, Level Set Methods and Fast Marching Methods, second edition Edition, Cambridge University Press, 1999.

[25] H. Flanders, Differentiation Under the Integral Sign, The American Mathematical Monthly 80 (6) (1973) 615-627. doi:\{10.2307/2319163\}.

[26] S. Whitaker, The Method of Volume Averaging, Theory and Applications of Transport in Porous Media, Springer Netherlands, 1998.

[27] R. I. McLachlan, H. Segur, A note on the motion of surfaces, Physics Letters A 194 (3) (1994) 165 - 172. doi:http://dx.doi.org/10.1016/0375-9601(94)91278-5.

[28] Hernderson, A., ParaView Guide, A Parallel Visualization Application, Kitware Inc. (2007). 
[29] Q. Krol, H. Löwe, Analysis of local ice crystal growth in snow, J. Glacio]doi:10.1017/ jog.2016.32.

[30] R. L. Shreve, Migration of air bubbles, vapor figures, and brine pockers in ice under a temperature gradient, J. Geophys. Res. 72 (16) (1967) 4093-4100.

[31] F. Flin, J.-B. Brzoska, B. Lesaffre, C. Coléou, R. A. Pieritz, Three-dimensional geometric measurements of snow microstructural evolution under isothermal conditions, Ann. Glaciol. 38 (1) (2004) 39-44. doi:10.3189/172756404781814942.

[32] T. Theile, H. Löwe, T. Theile, M. Schneebeli, Simulating creep of snow based on microstructure and the anisotropic deformation of ice, Acta Materialia 59 (18) (2011) 7104 - 7113. doi:http://dx.doi.org/10.1016/j.actamat.2011.07.065.

[33] S. Schleef, H. Löwe, M. Schneebeli, Influence of stress, temperature and crystal morphology on isothermal densification and specific surface area decrease of new snow, The Cryosphere 8 (5) (2014) 1825-1838. doi:10.5194/tc-8-1825-2014.

[34] H. Garcke, Curvature driven interface evolution, Jahresbericht der Deutschen Mathematiker-Vereinigung 115 (2) (2013) 63-100. doi:10.1365/s13291-013-0066-2.

[35] N. Calonne, F. Flin, B. Lesaffre, A. Dufour, J. Roulle, P. Puglise, A. Philip, F. Lahoucine, C. Geindreau, J.-M. Panel, S. Rolland du Roscoat, P. Charrier, CellDyM: A room temperature operating cryogenic cell for the dynamic monitoring of snow metamorphism by time-lapse X-ray microtomography, Geophys. Res. Lett. 42 (10) (2015) 3911-3918. doi:10.1002/2015GL063541.

[36] B. R. Pinzer, M. Schneebeli, Snow metamorphism under alternating temperature gradients: Morphology and recrystallization in surface snow, Geophy. Res. Lett. 36 (23) (2009) L23503, 123503. doi:10.1029/2009GL039618. 
Figure 1: Idealized grains (spheroids A,B,C and torus) used to assess the impact of surface smoothness on the accuracy of Eq. 20 for a constant velocity. The surfaces are colored by mean curvature $H$.

Figure 2: Scatter plots of measured and modeled rates from Eq. 20 for interface parameters of the grains from Fig. 1] For a torus $\bar{K}$ (and therfore $\dot{\bar{K}}$ ) is exactly zero (not included) which in turn implies $\dot{s}$ to be constant.

Figure 3: Cubic cutouts of the ice-air interface at $t=0$ (left) and $t=288 h$ (right) from Series 1 (top, cube length $1.26 \mathrm{~mm}$ ) and Series 2 (bottom, cube length $2.52 \mathrm{~mm}$ ) to demonstrate the morphological differences between initial and late stage. The interfaces are colored by the estimated normal velocity $v_{n}$.

Figure 4: Comparison of measured and modeled rates for the parameters $\phi, s, H, K, \chi$ and $H^{2}$ for two metamorphism time series. Solid lines correspond exactly to Eq. 200 and dashed lines to the sensitivity run where the surface gradient terms are neglected.

Figure 5: Comparison of measured and modeled evolution of $\dot{s}$ according to 22 to assess the growth law Eq. 21].

Figure 6: Evolution of $\bar{H}$ and $\overline{H^{2}}$ restricted to growing $\left(n_{z}<0\right)$ and sublimating $\left(n_{z}<0\right)$ parts of the interface to demonstrate the kinetic asymmetry in the growth law. 

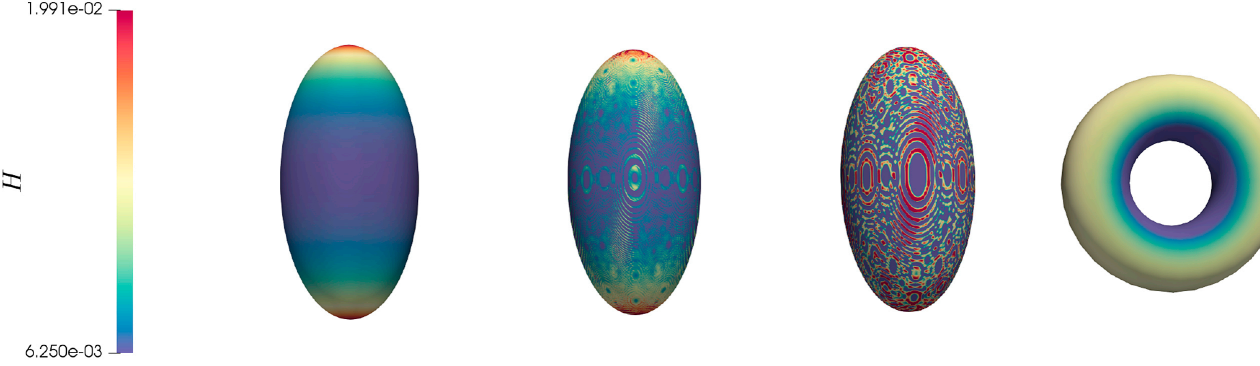

spheroid A

spheroid B

spheroid C torus 

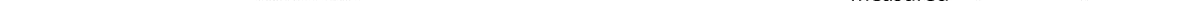

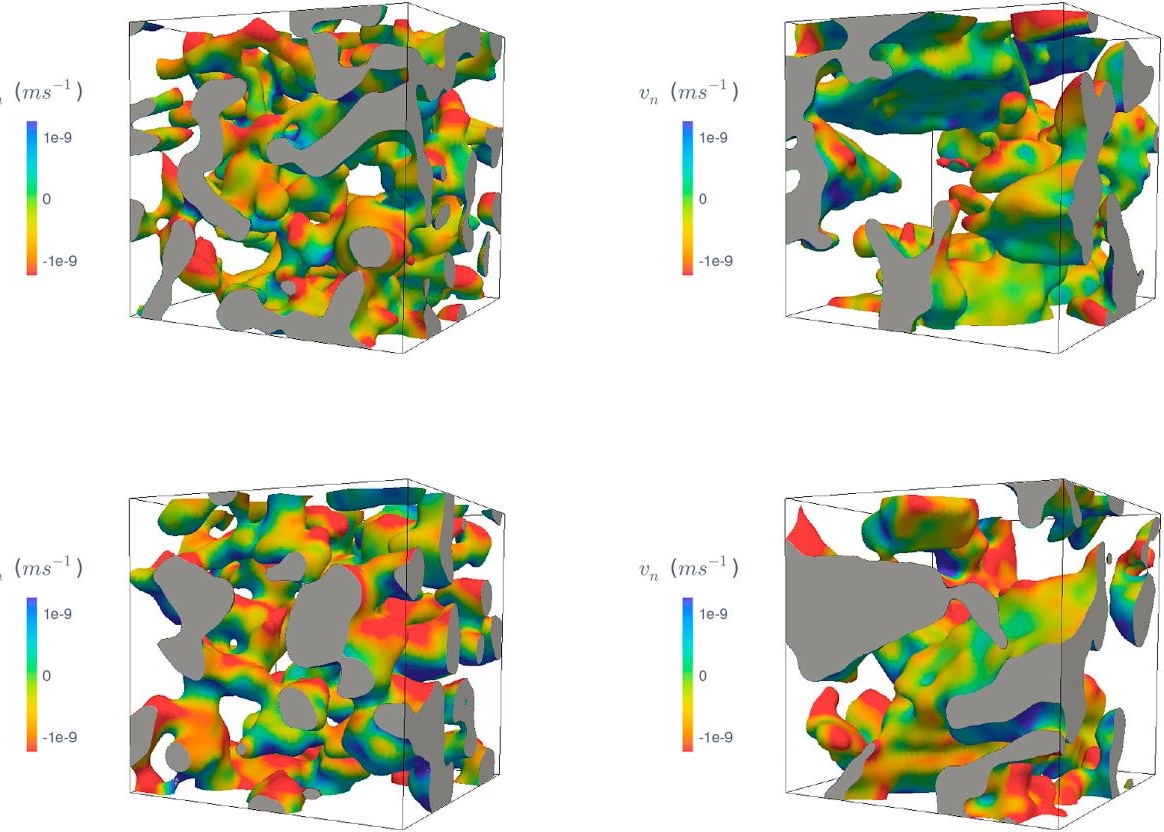


\section{$\times 10^{-3}$}

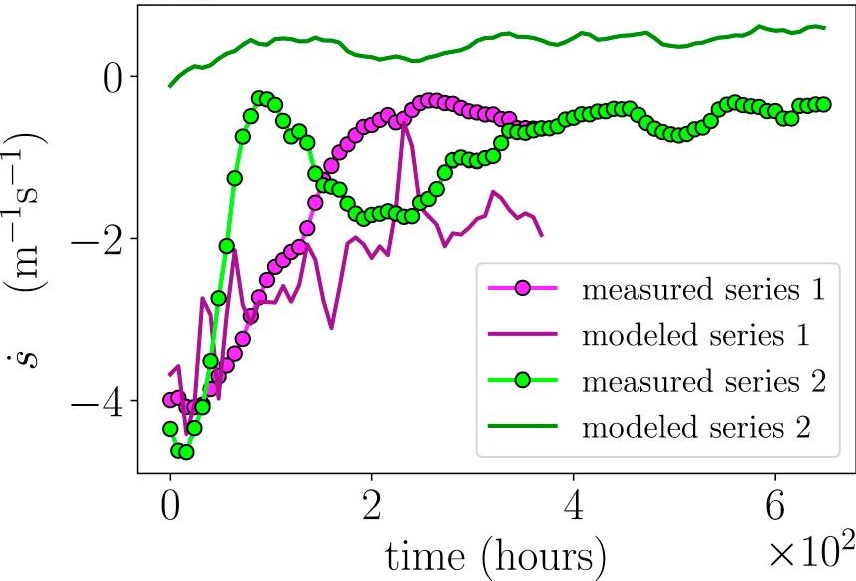




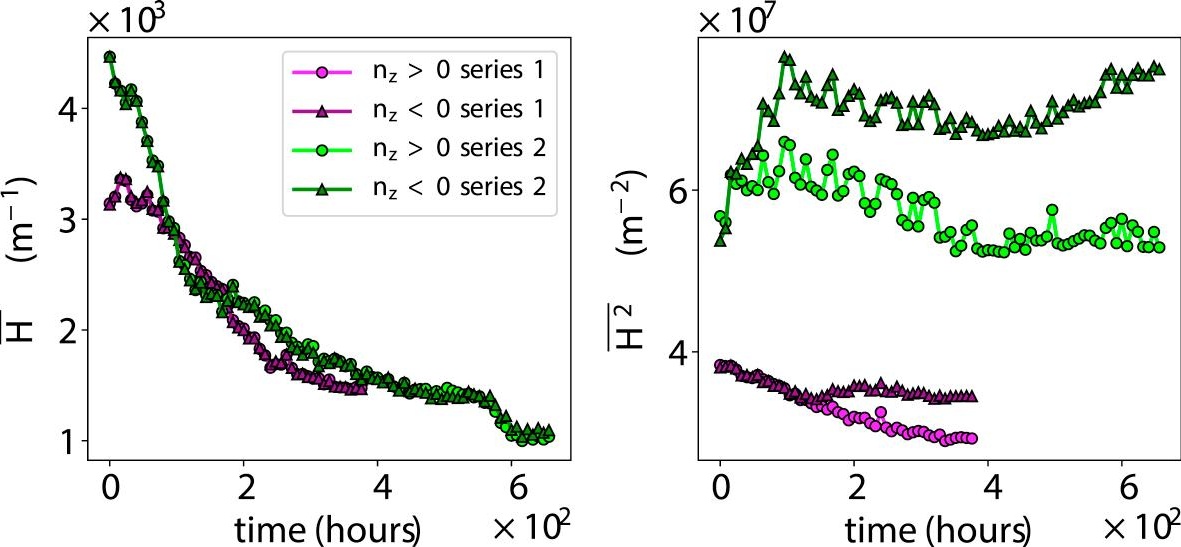


